\title{
Observations sur P'écologie et la distribution des Turbellariés Tricladides dépigmentés et anophtalmes de Roumanie
}

\author{
par
}

\author{
L. BOTOSANEANU*
}

\begin{abstract}
SUMMARY
Ecological and distributional remarks on unpigmented and anophthalmous Turbellaria Tricladida in Romania
\end{abstract}

22 localities where unpigmented and mostly blind tricladid turbellarians (Dendrocoelidae and Fonticola) were discovered by the author himself, are described in more or less detail. These animals are particularly well represented in Romania; the explanation is that they are expansive offshoots of a fauna formely inhabiting the huge brackish or freshwater lakes which covered most of this country during the Neogene (and especially the Sarmatian). Different species are inhabitants of different particular habitats of the underground water realm, and the author distinguishes between species inhabiting cave waters, typical phreatobionts, hyporheic species and species living in springs or springbrooks. These species are sensitive indicators of even small changes affecting the abiotic or biotic conditions prevailing in their habitats (several examples are offered, especially of competitive exclusion).

„Good ecological research is sooner obtained by individual enterprise than with computerized programmes"

L. B. Holthuis

En ces 25 dernières années j'ai eu souvent l'occasion - lors de recherches hydrobiologiques, spéléologiques et entomologiques réalisées un peu partout en Roumanie - de découvrir des stations à Tricladides dépigmentés et très souvent anophtalmes. L'observation, riche d'enseignements, de ces remarquables animaux obscuricoles et de leurs habitats très divers, m'a toujours passionné; je trouve donc qu'il serait utile d'essayer de faire maintenant le point. Plusieurs remarques préliminaires s'imposent. J'ai assez souvent fait des récoltes de ces animaux dont la fixation et la conservation convenables posent pas mal de problèmes; plusieurs espèces ont ainsi pu être décrites, mais des exemplaires en provenance d'autres stations découvertes par moi, restent non étudiés.

* Limnologische Flußstation, 6407 Schlitz, B.R.D. 
Je dois souligner que des Tricladides obscuricoles ont été découverts, dans de nombreuses autres stations de Roumanie, par des collaborateurs de la BIOSPEOLOGICA (descriptions par de Beauchamp), par un spécialiste comme le Prof. R. Codreanu et par plusieurs collaborateurs de l'Institut de Spéologie "E. Racovitza" (descriptions par R. Codreanu \& D. Balcesco). Mais j'ai l'intention de m'en tenir ici à mes propres découvertes et observations sans toutefois faire référence aux stations hyporhéiques dont je n'ai jamais fixé d'exemplaires. Dans ce qui suit, les stations sont énumérées dans l'ordre suivant: Mts. du Maramures et nord des Carpates Orientales (I-III); Carpates Méridionales, d'Est en Ouest (IV-XVI); monts calcaires du Banat (XVII-XXI); Plaine Occidentale (XXII).

I. Source rhéocrène tributaire d'un ruisselet à son tour affluent du ruisseau Sasar dans son cours supérieur, près de la route Suior-Baia Sprie et à peu près au même niveau que le chalet touristique Mogosa (Mts. du Maramures). Alt. approx. 700-800 m. Forêt de hêtres, zone éruptive. Le 14.XI.1962 le débit est insignifiant, dans le lit étroit et long de plusieurs dizaines de mètres, l'eau sort au jour sur les derniers mètres seulement, et fort discrètement. Température à la source même: $7,5^{\circ} \mathrm{C}$. Substratum représenté par des pierres anguleuses recouvertes d'Hépatiques, et reposant sur du limon. Sous toutes les pierres, nombreux Dendrocoelides blancs, et aussi des Niphargus; fort lucifuges, les vers disparaissent dans le substratum aussitôt qu'une pierre a été détachée du lit. Le Dendrocoelide a été fixé mais pas encore étudié; il pourrait s'agir éventuellement (inf. verb. Prof. R. Codreanu) de Dendrocoelum carpathicum Komar.

II. Niche madicole, dans la vallée Runcului dans son cours supérieur, à $2 \mathrm{~km}$ env. en aval de Podu Ciresului (Mts. du Maramures, massif Gutin). A $700 \mathrm{~m}$ d'alt environ, en forêt de hêtres. Le 23.v.1964, observé un tricladide (Dendrocoelide? Fonticola?) dépigmenté et aveugle, glissant sur la paroi suintante de roche cristalline. Je n'ai pas conservé d'exemplaires.

III. Source dans la vallée de la Bistrita, près de la route Cirlibaba-Borsa, entre Prinos et Sesuri ("à quelques m de la borne du $\mathrm{km} \mathrm{48,} \mathrm{à} 30 \mathrm{~m}$ de la route", sur le talus boisé - forêt d'épicéas). Nord des Carpates Orientales. Il s'agit de plusieurs filets d'eau à peine perceptibles. Le 28.V. 1953 j'avais noté "Planaires blanches et $C$. alpina partout en masse" ( 2 jours auparavant j'avais noté quelque chose de similaire pour un limnocrène au bord du grand ruisseau Lala, affluent de la Bistrita). J'ai maintenant des doutes: s'agissait-il vraiment de Tricladides dépigmentés? Il faudrait revoir ces stations.

IV. Le cours inférieur du ruisseau Cumpenita à Cumpana A rgesului (Carpates Méridionales, versant $\mathrm{S}$. du massif de Fagaras); parfois désigné sous le nom de Valea Topologului, ce qui est certainement erroné. C'était un tributaire du grand ruisseau Capra; les deux se jettent maintenant directement dans un grand lac d'accumulation. Alt. $770 \mathrm{~m}$ env., zone de la forêt de hêtres, roches cristallines. Mes observations datent des 10-13.IX.1957, 13.VI.1961, 9.IX.1961, 13.X.1961, 3.XI.1961, et ont été réalisées sur les 2-3 km inférieurs du cours de la Cumpenita, dont les eaux s'écoulent rapidement ou très rapidement dans un radier rempli de pierres moyennes ou grosses. Largeur: $10-12 \mathrm{~m}$ en période de 
crues, autrement beaucoup moindre; profondeur - en automne $-30-40 \mathrm{~cm}$ en moyenne; température bien entendu fort variable. En arrachant du radier, parfois avec beaucoup de peine, de grosses pierres bien implantées, et ceci surtout - mais pas uniquement - près des bords du ruisseau et en eau s'écoulant rapidement, j’ai constaté la présence sur leurs surfaces inférieures, d'exemplaires d'un grand et beau Dendrocoelide dépigmenté et anophtalme, fragile, à bords festonnés. Certains endroits fournissaient des exemplaires, nombreux ou isolés, presque sous chaque pierre, dans les circonstances mentionnées; pas un seul exemplaire dans d'autres endroits. Le 13.VI.1961 et le 3.XI.1961 mes efforts restèrent vains. Les exemplaires recueillis ont permis la description de Dendrocoelum (Polycladodes) affine Codreanu \& Balcesco, 1970 (v. aussi station suivante, les deux sont les localités typiques de l'espèce).

V. "Valea cu Pesti", dans son cours inférieur, à approx. $700 \mathrm{~m}$ d'alt., dans la forêt mixte - épicéas et hêtres (Carpates Méridionales, versant S. du massif de Fagaras). Ce grand ruisseau était un affluent gauche du Arges à $3 \mathrm{~km}$ environ en amont de ses Gorges; il se jette maintenant dans le grand lac d'accumulation ci-dessus mentionné, dont les eaux couvrent actuellement la zone où j'avais découvert les Dendrocoelides. Grand ruisseau, à fort débit même en période de sécheresse, largeur 2-3 m, pente modérée, cours habituellement rapide (grands blocs, pierres petites et moyennes), plus rarement calme (sable, gravier). Le 11.IX.1961 j’y ai découvert un Dendrocoelide dépigmenté et anophtalme, décrit ensuite sous le nom de Dendrocoelum (Polycladodes) affine Codreanu \& Balcesco, 1970; on le trouve exclusivement sous les pierres moyennes ou grandes, puissamment ancrées dans le substratum de sable et de gravier. Il faut chercher longtemps avant de trouver un bon endroit; ces "bons endroits" se signalent toujours par l'aspect marécageux (suintant) des zones bordant le ruisseau (affleurement du sous-écoulement).

VI. Complexe de sources rhéocrènes au lieu dit "La Rezervor", dans les Gorges du Arges, alt. $650 \mathrm{~m}$ environ, à plusieurs $\mathrm{km}$ en aval donc de la station précédente. Le 12.IX.1957 j'avais noté la présence, en grand nombre et à côté de C. alpina (!), sur les fragments ae gneiss qui tapissent le lit de ces rhéocrènes, d'un "remarquable Polycelis d'un blanc-laiteux, à cornes pointues, noires tout comme la limite frontale". Pas de matériel conservé. Il n'est pas du tout sûr qu'il s'agisse vraiment d'un Polycelis, mais c'est un Tricladide dépigmenté qu'il faudrait essayer de retrouver (si toutefois la station a survécu aux travaux hydroénergétiques réalisés dans la zone).

VII. Le 17.VI.1955 j'avais noté la présence d'une "planaire blanche" dans un petit ruisseau sur forte pente, affluent droit du Olt, tout près de la route Sibiu-Calimanesti qui longe la rivière dans son Défilé, à quelques $\mathrm{m}$. en aval de la source captée et murée, connue sous le nom de Izvoru Imparatului ou Kaiserbrunnen (à $2,5 \mathrm{~km}$ en aval de la commune de Boita, alt. approx. $350 \mathrm{~m})$. J'ai revu la localité le 17.XI.1977, et j'ai constaté que des Fonticola dépigmentées et anophtalmes habitent plusieurs petits cours d'eau situés sur la même pente. Le ruisselet visité en 1955 restait pratiquement inchangé, mais je l'ai laissé de côté, mon attention ayant été attirée par 2 ruisselets de source, situés à $700 \mathrm{~m}$ environ en aval de la "Kaiserbrunnen", au lieu dit "La 
Ograda". Un de ceux-ci est long seulement de $50 \mathrm{~m}$, il parcourt un pré sur forte pente et pratiquement dépourvu d'arbustes, mais à sa source frontale (aménagée par l'homme en bassin limnocrène) il y a un aulne; le bassin limnocrène, avec du limon et quelques galets sur son fond, est suivi par $1 \mathrm{~m}$ de ruisselet fort piétiné, mais le tronçon de $30 \mathrm{~m}$ qui fait suite représente un habitat resté à l'état naturel et dont la végétation (mousses, Carex, etc.) montre qu'il est toujours en eau; sur le substratum de sable se trouvent des pierres et des galets, mais ceci seulement sur les 20 premiers $\mathrm{m}$; le tronçon décrit est à son tour suivi par un autre, long de $20 \mathrm{~m}$, où le lit est totalement envahi par l'herbe et l'eau disparaît presque entièrement (aspect tout simplement de "pré humide"); débit fort modeste, et ceci sans doute de façon permanente, mais le cours garde une certaine vivacité grâce à la forte pente; largeur du lit: $10-30 \mathrm{~cm}$, profondeur de l'eau infime, temp. $11,5-12^{\circ} \mathrm{C}$ le 6.XI.1977, pas de Tricladide pigmenté dans la faune. Dans le limnocrène et le premier $m$ du ruisselet, inutile de chercher des Fonticola; celles-ci (espèce de petite taille, à mon avis probablement nouvelle; matériel vivant collecté le 6.XI.1977, par le Prof. R. Codreanu et Mme. D. Balcesco-Codreanu) habitent les $10-15 \mathrm{~m}$ du ruisselet qui font suite au court tronçon piétiné; observation intéressante: sous la plupart des pierres les Gammares foisonnent littéralement, et dans ces conditions il n'y a pas de Fonticola mais quand on soulève une petite pierre sous laquelle les Gammares sont peu nombreux et jeunes, ou même absents, les Fonticola font leur apparition (parfois 10 exemplaires sous un petit galet); plusieurs exemplaires ont été vus circulant à découvert sur le sable fin; dès que les pierres disparaissent du lit, il devient impossible d'en trouver. Un autre ruisselet, nettement plus long que le premier et à débit un peu plus important, est situé à quelques dizaines de $\mathrm{m}$ en amont, sur la même pente, et parcourt une zone couverte par un dense taillis; sous les pierres, nombreuses Planaires pigmentées (Dugesia) mais pas de Fonticola dépigmentés; celles-ci se montrent, par contre, nombreuses dans d'infimes sources latérales alimentant le ruisselet (aspect de hélocrènes, sortie extrêmement discrète de l'eau, galets humides couverts de mousses, faune plutôt madicole, sans planaires pigmentées).

VIII. Ruisseau Bucureasa, assez important tributaire droit du Lotru, à $1 \mathbf{~ k m}$ en amont du centre du village Malaia (Carpates Méridionales, Mts. de LotruCapatîna, alt. approx. $520 \mathrm{~m}$ ). En travaillant tout près du confluent, le 1.IX.1952 j'ai trouvé 2 exemplaires (malheureusement non conservés) d'un Tricladide blanc et aveugle "sous une pierre ancrée juste dans la berge du ruisseau et bien humectée par l'eau". Le 8.IX.1977 cette station (les $100 \mathrm{~m}$ inférieurs du cours du ruisseau) était pratiquement anéantie par des travaux en cours (construction d'un barrage); un peu plus en amont j'ai vainement tenté, en arrachant des pierres du radier, de retrouver le Tricladide.

IX. Source limnocrène toute petite, formant un ruisselet minuscule et à débit infime, du côté gauche de la Bahna (Mts de Mehedinti, alt. $430 \mathrm{~m}$ ), à une certaine distance en amont du confluent de ce grand ruisseau avec le Pîrîu Gornentilor. Le 22.V.1957 j'y ai trouvé des "Planaires blanches" que je n'ai pas pu fixer et conserver.

X. Dans le massif de Domogled, séparant la vallée de la Cerna de celle de la 
Bahna, et appartenant aux Mts de Mehedinti, sur le sentier reliant Baile Herculane au village de Podeni, à $2 \mathrm{~km}$ environ à partir de la source "Izvoru Jalarau" en direction de Podeni, dans une zone calcaire à caractère de plateau boisé (hêtres) et légèrement vallonné, j’ai été, le 21.V.1957, après une période prolongée de pluies torrentielles, témoin d'un remarquable phénomène (voir aussi: Botosaneanu, 1971). Dans un endroit normalement à sec, par suite de la pression hydrostatique considérablement accrue, l'eau jaillissait en de nombreux endroits du sol de la forêt de hêtres; sur une zone longue de $100 \mathrm{~m}$ environ et large de 5 à $20 \mathrm{~m}$ le sol de la forêt ainsi que la route étaient inondés par cette eau à $7-9^{\circ} \mathrm{C}$, et il s'individualisait rapidement un ruisselet, s'unissant à un autre venu d'un peu plus loin, l'eau aboutissant à "Izvoru Jalarau" précédemment mentionné. Le ruisselet s'écoule sur pente assez aide; dans son "lit"; sable, gravier menu, amas de feuilles mortes. Il était possible d'observer glissant un peu partout sur le sable et les cailloux, et aussi dans les sillons remplis d'eau et tracés dans la boue par les roues des chars à boeuf, de nombreux exemplaires d'une Némerte obscuricole, et de très nombreux grands Dendrocoelides blancs, fort agiles; le matériel conservé, soumis à un examen préliminaire par le Prof. R. Codreanu, a permis de conclure (inf. verb.) qu'il s'agit d'une espèce fort voisine du Dendrocoelum (Paradendrocoelum) spelaeum (Kenk) décrit de Slovénie. Deux jours plus tard (23.V.1957), les pluies torrentielles se poursuivant, le ruissellement superficiel avait pratiquement anéanti cet habitat précaire. J'ai essayé à plusieurs reprises, surtout après des périodes pluvieuses prolongées, de retrouver cet habitat, mais en vain: il était à sec. Cependant, le 29.VI.1961, toujours après une période prolongée de fortes pluies, j'ai constaté que le ruisseau qui m'avait fourni Dendrocoeles et Némertes en mai 1957, n'existait toujours plus, tandis que l'autre, venant d'un peu plus loin dans la forêt (long de $150 \mathrm{~m}$ environ, large de $20 \mathrm{~cm}$, à eau s'écoulant vivement sur lit à aspect de rigole et rempli de gravier; $t=9^{\circ} \mathrm{C}$, "source frontale" changeant visiblement d'emplacement), m'a fourni des Dendrocoelides assez nombreux (ainsi qu'un Niphargus, mais pas de Némertes); les Dendrocoelides sont présents seulement en certains endroits, et seulement de 10 à $40 \mathrm{~m}$ en aval de la source actuelle; ils deviennent plus nombreux si l'on remue le gravier; ils me semblent être plus petits, plus graciles, plus allongés que ceux de 1957 (le 29.IX.1966, ce même ruisselet - bien que toujours en eau - ne contenait plus de Dendrocoelide!).

XI. Deux sources sur la rive gauche de la Cerna, approximativement au $\mathbf{k m}$ 13,5 sur la route en amont de Baile Herculane, au lieu-dit "La Bulza". Débit modeste, $\mathrm{t}=9^{\circ} \mathrm{C}, \mathrm{pH}=6,5$. Des Dendrocoelides obscuricoles avaient été trouvés le 14.X.1957, en exemplaires peu nombreux, dans les deux sources (dans une de celles-ci, petite source karstique sur forte pente et à lit rocheux revêtu de mousses, connue sous le nom de "Sipotu lui Niculae", ils coexistaient apparemment avec Polycelis cornuta). Pas de matériel conservé. Le 3.IX.1977, les 2 sources étaient complètement taries depuis 3 semaines (automne pourtant pas particulièrement sec) et dans de petites sources à proximité il n'y avait que des Crenobia alpina.

XII. Un des petits complexes de sources frontales (plus exactement: celui du 
côté droit dans le sens de la montée!; dans celui du côte gauche, situé considérablement plus en haut de la même pente, je n'ai jamais vu des Tricladides dépigmentés) alimentant le "Ogasu UImului", petit affluent gauche du ruisseau Frasincea - un des bras du cours supérieur de la Belareca, bassin et Mts de Cerna; village Frasincea, commune de Cornereva; alt. $1000 \mathrm{~m}$ environ. Voir aussi Botosaneanu, 1971. L'eau sortait au jour (en 1957), de nombreuses fissures et en 2 endroits, au pied d'une paroi assez haute, en forêt de hêtres. Les 2 sources, à débit peu important, avaient aspect de limnocrènes; $t$ variant de 6,5 à $8,3^{\circ} \mathrm{C}$ (fin avril — début septembre), $\mathrm{pH}=5($ !); sur le fond, du sable, des fragments de pegmatite et - surtout au printemps et en automne - des amas de feuilles mortes imbibées d'eau; le ruisselet alimenté par ces sources (long de quelques dizaines de $\mathrm{m}$, à débit relativement grand et dont l'eau s'écoule sur pente relativement raide, tandis que la pente est très raide pour le Ogasu Ulmului après le confluent des ruisselets de source) a dans son lit des pierres pegmatite - recouvertes de mousses, et il est au printemps littéralement enseveli sous d'épaisses couches de feuilles mortes; lorsque la température était de $8^{\circ} \mathrm{C}$ à la source, elle atteignait $10,5^{\circ} \mathrm{C} 25 \mathrm{~m}$ plus bas. Dans ce petit complexe de sources eustatiques et sténothermes, l'association faunistique est dominée par des crénobiontes, mais les éléments obscuricoles y sont représentés par un Niphargus du gr. foreli, par des Rhabdocoeles, et - je répète, seulement dans les sources du côté droit, sens de la montée - par une grande population de Dendrocoelides. J'y ai fait des observations le 30.IV.1957, le 1.VIII.1957, le 16.X.1957, pour y revenir 20 ans après. Les Dendrocoelides Dendrocoelum (Dendrocoelides) debeauchampianum Codreanu \& Balcesco, 1967 - y étaient régulièrement trouvés en masse (jusqu'à 30 exemplaires sous une petite pierre!) dans les fissures d'où sourd l'eau, dans les limnocrènes, et seulement dans la zone initiale ( 10 m environ) du ruisselet; on les trouvait sous les pierres - surtout petites - entre les feuilles mortes; extrêmement lucifuges, ils disparaissaient rapidement lorsque la couverture de branchages et de feuilles était éliminée. J'ai noté aussi que dans des ruisselets similaires situés à proximité, il n'y avait aucune trace de Dendrocoele. Le 1.IX.1977: dans les sources du bras gauche toujours pas de Dendrocoeles; des 2 sources alimentant le bras droit, celle supérieure - située juste au pied de la paroi - était tarie; la seconde, à débit fort amoindri et assez endommagée par piétinement $\left(\mathrm{t}=8,3^{\circ} \mathrm{C}\right)$ abritait une population de Dendrocoeles nettement appauvrie par rapport à celle remarquée 20 ans avant.

XIII. Deux sources limnocrènes connues sous le nom de "Izvoru Rau", toujours à Frasincea-Cornereva (v. st. XII). Du gravier dans l'une des sources, des feuilles mortes dans la 2ème, eau à $9^{\circ} \mathrm{C}$ le 30.IV.1957. Des Dendrocoeles, fort probablement $D$. debeauchampianum, y ont été trouvés, mais en nombre limité. Et j’ai noté que, dans d'autres sources en proximité immédiate, les Dendrocoeles obscuricoles étaient remplacés par des Tricladides pigmentés.

XIV. 2 autres sources dans la même zone que st. XII et XIII (mes notes ne permettent pas une localisation satisfaisante). $\mathrm{T} .=8.5^{\circ} \mathrm{C}$ (1.VIII.1957), pas de végétation, pierraille fort meuble. J'ai noté la présence de "Planaires blanches extrêmement rares" (certainement la même espèce que pour XII et XIII). 
XV. La source de la bergerie "In Povoiu Rosu" (="Izvoru Povoiului"), tributaire du cours supérieur de la Iauna, important affluent droit de la Cerna dans le cours supérieur de cette rivière (Mts. de Cerna). La Iauna prend ses sources dans un cirque glaciaire ("Fata Iablaniceanului") à 1200-1400 m d'alt. environ, au dessus de la limite supérieure de la forêt; notre source se trouve un peu plus bas, en pleine forêt de hêtres (1100-1200 m d'alt. probablement); accessible soit à partir de la commune de Cornereva, soit à partir du confluent Cerna-Iauna. C'est un fort rhéocrène, à débit considérable; la source frontale change visiblement d'emplacement en fonction du débit; lit rempli de pierres, $\mathrm{t}=5,3^{\circ} \mathrm{C}$ en plein été. Le 2.VIII.1957 j'ai observé et récolté sous les pierres, dans le secteur initial du rhéocrène de nombreux Dendrocoelides obscuricoles de taille assez considérable. Malheureusement ce matériel s'est égaré. Il n'est pas exclu qu'il s'agisse de la même espèce que dans st. XVI.

XVI. 2 sources rhéocrènes (voir aussi: Botosaneanu, 1971) situées dans le massif Muntele Mic, à 1350-1400 m d'alt. environ (l'altitude la plus considérable à laquelle un Tricladide dépigmenté et anophtalme ait jamais été découvert en Roumanie!). Elles se trouvent tout près du sentier touristique reliant Poiana Marului aux châlets de Muntele Mic (via Pietrele Scorilei), en pleine forêt mixte et en zone entièrement métamorphique, avant que le sentier ne débouche dans la clairière "Poiana Boboratei". Station découverte le 17.VI.1970, visitée à nouveau le 30.IX.1970 et le 6.IX.1977. Mes observations et récoltes ont été réalisées, lors des 3 premières visites, surtout dans le rhéocrène du côté droit (sens de la montée); dans l'autre, des Dendrocoelides obscuricoles avaient été observés, mais leur densité semblait être moindre (peut-être à cause de la présence d'une importante population d'un Niphargus du gr. stygius -carpathicus). Il s'agit de rhéocrènes typiques, à débit modeste mais à cours agreste même en période de sécheresse; dans leur lit étroit: sable, gravier, pierres, débris végétaux; le 30.IX.1970 l'eau était à $5,2^{\circ} \mathrm{C}$ à partir de la source frontale et jusqu'à 12-13 m en aval. La population de Dendrocoeles obscuricoles était extrêmement importante; la densité atteignait un maximum entre la "tête" du rhéocrène et un point situé à quelques $\mathrm{m}$ en aval; on trouvait ces animaux jusqu'à $15 \mathrm{~m}$ en aval, mais ensuite ils disparaissaient brusquement. Quelques observations d'un certain intérêt ont pu être faites: une grande pierre plate ayant été extraite de la source, un très grand nombre de Dendrocoeles ont commencé à surgir du substratum dans lequel cette pierre était implantée; tout près de la "tête" du rhéocrène les animaux sont généralement juvéniles, et c'est plus en aval (jusqu'à $15 \mathrm{~m}$ ) qu'on rencontre les exemplaires matures; peu en aval du point de la sortie de l'eau, de nombreux exemplaires ont été observés circulant à découvert sur le sable ou sur le menu gravier; tous ces exemplaires montraient un rhéotactisme positif très net. Le 6.IX.1977, alors que dans la source du côte gauche (sens de la montée) la situation restait inchangée (assez nombreux Dendrocoeles dans la source frontale et sur 1,5 $\mathrm{m}$ de ruisselet lui faisant suite, sous les pierres et les petits galets, mais aussi sur le substratum sous-jacent de fin limon végétal et de sable), dans celle du côté droit de légères modifications physiographiques ("aménagement" par les passants d'un petit "bassin limnocrène" juste au point de sortie de l'eau), avaient entrainé 
une baisse apparemment considérable du nombre de ces vers, qui sont maintenant isolés, tandis que Crenobia alpina est devenue abondante un peu plus en aval sur le ruisselet. Une grosse pierre ayant été arrachée du sol près de la source frontale, dans un endroit apparemment sans eau, un mince filet d'eau phréatique s'est fait jour, et il y avait un Dendrocoele dépigmenté sur la face humide de la pierre. Toutes les observations montrent qu'il s'agit d'animaux qui sortent du phréatique pour s'installer, de façon précaire, dans des secteurs fort limités des sources. Il est vraisemblable que l'espèce est inédite (abondant matériel conservé).

XVII-XIX. Trois grottes horizontales groupées sur une superficie assez restreinte de la grande plate-forme de calcaires mésozoïques, connue sous le nom de "zone Resita-Moldova Noua" (mts. du Banat, plus exactement Mts. d'Anina, entre les vallées de la Nera et du Caras). Ces trois grottes, ainsi que leurs habitats aquatiques, ont été décrites de façon suffisamment détaillée dans Botosaneanu, Negrea et Negrea (1967) et respectivement dans Botosaneanu (1971); ce sont: Pestera Marghitas, Pestera Tolosu, Pestera Ponor-Plopa. Leurs eaux m'avaient fourni (1961-1966) des exemplaires, parfois nombreux, parfois isolés, d'un Dendrocoelide souterrain qui a été décrit sous le nom de Dendrocoelum (Eudendrocoelum) botosaneanui del Papa (del Papa, 1965, 1970)*. Je me résume ici à citer quelques lignes dédiées à cette espèce dans Botosaneanu (1971): "Le seul Triclade des grottes des Monts du Banat connu à ce jour est Dendrocoelum (Eudendrocoelum) botosaneanui del Papa... Nous avons capturé cette belle espèce... dans trois grottes... Dans les trois cas il s'agit de grottes sub-actives ou bien de galeries sub-actives de grottes plus complexes. Plus exactement, D. botosaneanui a été capturé dans des laisses d'eau de ruisseaux souterrains ("lacs", marmites et autres formes négatives du plancher remplies d'eau rémanente): il est donc clair qu'il s'agit d'un vrai habitant des cours d'eau souterrains. D'ailleurs, nous l'avons capturé même dans des eaux vives dans Pestera Tolosu, où il peuple un ruisselet endogène. On peut parler d'une préférence pour le substratum argileux, mais le substratum sableux n'est pas évité. Des observations ont pu être faites sur la survie en période de sécheresse (le 27.IX.1965, dans la Pestera Tolosu, dans une marmite à sec le dépôt de sable couvert par une croûte argileuse était encore humide; en enlevant des morceaux d'argile, on trouve en dessous des $D$. botosaneanui immobiles, contractés, mais bien vivants, qui survivront jusqu'à la remise en eau... Les populations de P. Tolosu et de P. Ponor-Plopa sont sans doute importantes, mais nous n'avons pas le droit d'affirmer que celle de P. Marghitas ne l'est pas: il ne faut pas oublier que nous n'avons jamais utilisé des pièges appâtés!...". Les Dendrocoeles ont été trouvés en compagnie d'une faunule de troglobies: l'Oligochète Pelodrilus bureschi Mich., l'Harpacticoïde

*) En 1977, un groupe de spéléologues amateurs de Resita (inf. G. Karban et M. Tigla) a remarqué la présence de Tricladides blancs (presque certainement l'espèce ci-dessus mentionnée) "dans des gours" situés au fond de deux avens (Avenul Mare de la Pauleasca et Avenul de la Raspıntii), dans un de ceux-ci à $-54 \mathrm{~m}$. Ces deux avens sont creusés dans la même zone que les 3 grottes citées. 
Maraenobiotus brucei carpathicus Chapp., ainsi que Niphargus maximus (Kar.) et $N$. kochianus Bate (ssp.?).

$\mathrm{XX}$. Source rhéocrène dans la Dépression de Carasova (Mts. calcaires du Banat, commune de Carasova, à quelques $\mathrm{km}$ au S. de Resita, alt. $200 \mathrm{~m}$ ). L'eau sort au jour tout près de la rive droite du petit ruisseau Sereniak (affluent du Caras), à quelques $m$ en amont d'une source captée et des maisons du village. Cette source fait partie d'un important "horizon de sources" qui d'étend sur plusieurs km: mais c'est seulement celle-ci qui nous a fourni des Dendrocoelides dépigmentés et anophtalmes, et c'est la stationtype de Dendrocoelum (Dendrocoelides) atriostrictum Codreanu \& Balcesco, 1967 (voir aussi: Gourbault, 1967). Il n’y a pas d'autre élément obscuricole dans cette source. La station a été visitée aux dates suivantes: 5.X.1965, 1.X.1966, 6.X.1969, 13.VI.1970, 4.IX.1977. Elle n'est pas protégée par la forêt; le débit est assez petit mais constant; le substratum est représenté par un très grand nombre de fragments anguleux, grands ou petits, de calcaire, et il n'y a pas de végétation; la longueur de la source ne dépasse pas 3-3,5 m, et la température de l'eau varie de 11,6 à $12^{\circ} \mathrm{C}$ en automne $\left(10^{\circ} \mathrm{C}\right.$ au printemps). Le 5.X.1965 et le 1.X.1966, d'assez nombreux Dendrocoelides avaient été trouvés sous les pierres, pas tout à fait au point de sortie de l'eau, mais sur $1,5 \mathrm{~m}$ seulement du ruisselet, et jamais dans la zone tout à fait inférieure de la source. En 1969 la source avait été littéralement ensevelie sous un éboulis, à la suite de l'exploitation du calcaire à son voisinage immédiat; c'était seulement le secteur tout à fait inférieur de la source qui restait non enseveli, mais là je n'ai pu trouver de Dendrocoelides (même situation en 1970). Le 4.IX.1977, observations assez intéressantes; la partie frontale de la source était toujours ensevelie sous une masse de pierraille et de blocs, mais après avoir été partiellement désobstruée, elle a fourni des exemplaires isolés de $D$. atriostrictum; l'observation attentive de la rive droite du Sereniak, en amont et en aval de l'embouchure de la source, a révélé l'existence, sur $30 \mathrm{~m}$ environ, d'une série de fort discrètes sorties au jour de l'eau phréatique, du sol mélangé de pierraille et de sable et tout près du niveau de l'eau du ruisseau; il se forme ainsi une série de 4-5 minuscules sources longues de $30-60 \mathrm{~cm}$ à peine (est-ce une conséquence de l' "écrasement" de la source principale en 1969??); l'eau est à $11,7-11,8^{\circ} \mathrm{C}$; or, de nombreux Dendrocoeles ont été trouvés ici, pratiquement sous chaque pierre extraite de cet habitat à aspect assez précaire, et ceci renforce l'impression qu'il est question d'une espèce phréaticole trouvant seulement des "habitats secondaires" dans certaines sources.*

XXI. Résurgence représentant la source frontale du ruisselet Stîrmine, au lieu-dit "Cuptor", accessible sur un sentier, à quelques $\mathrm{km}$ du village Bradisoru de Jos (Maidan), dans les collines calcaires de la zone de Oravita (Banat). Alt. approx. $250 \mathrm{~m}$. Au lieu - dit mentionné il y a un énorme bloc de tuf

*) A quelques $\mathrm{km}$ à vol d'oiseau de cette station, dans un endroit sauvage des Gorges du Caras, au lieu-dit "La Teria", des spéléologues amateurs de Resita ont découvert - été 1977 - une source karstique abritant une population de Tricladides dépigmentés et aveugles (pas de matériel récolté); en septembre, la source était tarie et on n'a plus pu observer de Tricladides (inf. M. Tigla, S. Karban). Il est possible qu'il s'agisse toujours de D. atriostrictum. 
calcaire et c'est juste au-dessus de ce bloc, sur une sorte de petit plateau, que l'eau sort au jour, dans la forêt de feuillus et d'un chaos de blocs calcaires. Station visitée aux dates suivantes: 8.VII.1961, 7.X.1961, 29.IX.1966, 9.X.1969, 2.IX.1977. Tout le débit de la source frontale passe par un tuyau en métal, l'eau pénètre aussitôt dans la pierraille, pour ressortir - en période de sécheresse - à quelques dizaines de $\mathrm{m}$. en aval; là, dans une zone qui n'est plus protégée par la forêt, il se forme un ruisselet étroit et calme, avec - dans un secteur de $10 \mathrm{~m}$ environ, où il y a toujours de l'eau - beaucoup de pierres sur substratum de limon et de sable; l'eau est faiblement incrustante, ainsi que dans le secteur suivant du ruisselet, à eau fort calme sur substratum limoneux; ensuite le ruisselet dévale le gros bloc de travertin, et change complètement de caractère (eau fort incrustante, faune appauvrie et plus banale). Le débit est assez variable: en périodes de sécheresse accentuée, il n'y a plus d'eau dans le lit du ruisselet, en bas du bloc de tuf. Début septembre, l'eau à la source frontale est à $11^{\circ} \mathrm{C}$, et dans le secteur supérieur du ruisselet (celui à Dendrocoeles) à $12^{\circ} \mathrm{C}$; pratiquement invariable dans le premier cas, elle doit enregistrer des variations assez importantes dans le deuxième cas. Uniquement dans le secteur supérieur du ruisselet, long de $10 \mathrm{~m}$ (jamais dans la source frontale, jamais plus bas), une très importante population de Dendrocoelum (Dendrocoelides) banaticum Codreanu \& Balcesco, 1967 (voir aussi: Gourbault, 1967); c'est la localité type de cette espèce. On trouve les vers surtout sous les pierres, mais j'en ai remarqué aussi sur la face supérieure de celles-ci, ainsi que sur le substratum sous-jacent. Il existait un certain danger d'effondrement du bloc de travertin à cause de son exploitation intensive, mais ce danger semble temporairement écarté; en septembre 1977 j'ai remarqué que le piétinement par le bétail du secteur à Dendrocoeles avait déterminé une certaine régression de la population.

XXII. Lors d'une campagne de recherches, à l'aide du filet de Cvetkov, sur le peuplement des puits de la Plaine Occidentale de Roumanie, j'ai capturé, le 8.VI. et le 13.IX.1972, des Tricladides dépigmentés et anophtalmes dans plusieurs puits murés de la commune Satchinez - maisons aux Nos. 45, 592, 862 - à $100 \mathrm{~m}$ d'altitude et à $10 \mathrm{~m}$ de profondeur environ. Il s'agit d'animaux allongés, délicats; les exemplaires fixés n'ont pas encore fait l'objet d'une étude; ils ont été pris en compagnie d'une intéressante faunule phréaticole typique (Niphargus, Candona chappuisi Klie, Peloscolex zavreli Hrabe).

\section{QUELQUES CONCLUSIONS}

1. La faune de Tricladides dépigmentés et le plus souvent anophtalmes (essentiellement Dendrocoelidae et Fonticola) de Roumanie, même fort incomplètement connue comme elle l'est actuellement, s'avère être d'une richesse inouïe, et les stations peuplées par ces animaux sont très nombreuses et distribuées partout dans ce pays, indépendamment de la structure lithologique (celles dont il est question dans le présent travail représentent sans doute moins de la moitié de celles jusqu'à présent découvertes!). C'est donc un élément positif important dans la faune aquatique souterraine de Roumanie, faune en 
général nettement appauvrie par rapport aux zones méridionales d'Europe.

L'explication est du domaine de la paléogéographie. C'est pendant le Néogène, plus exactement au cours du Sarmatien, que l'extension - dans les limites de la Roumanie - de bassins à eaux saumâtres ou même tendant à s'adoucir, a atteint son maximum; au Sarmatien moyen et supérieur, un très important bassin pannonique occupant presque l'ensemble de la Transylvanie (sensu lato), était séparé d'un bassin Dacique tout aussi important, s'étendant au S. et à l'E. des Carpates, tandis que la majeure partie des Mts. Apuseni, des Mts. du Banat, des Carpates Méridionales et Orientales, représentait toujours des terres fermes. Ce sont vraisemblablement les eaux de ces grandes cuvettes lacustres qui abritaient les ancêtres de nos Dendrocoelides (obscuricoles ou non: car aux formes dépigmentées et dépourvues d'yeux s'ajoute une espèce de petite taille mais pigmentée et oculée, telle que Palaeodendrocoelum romanodanubialis Codr. du Danube). Lors du Pontien (surtout supérieur - Dacien), on assiste par contre à une spectaculaire régression des eaux du Bassin Pannonique, et c'est peut-être à cette époque que se place le début du peuplement en Dendrocoelides des réseaux hydrographiques des zones exondées autour de la chaîne des Carpates. Et c'est par les vicissitudes liées aux glaciations pléistocènes que l'on peut s'expliquer le fait de la persistance jusqu'à notre époque prèsque seulement de celles des espèces qui se sont réfugiées dans le domaine aquatique souterrain. Bien sûr, les détails de cette histoire et de cette remarquable prolifération ne seront jamais connus.

Plusieurs particularités régionales semblent pouvoir être reconnues: si des espèces phréaticoles proprement dites existent dans toutes les régions du pays, et si de vrais troglobies ont été découverts dans les grottes à eau de la plupart des zones karstiques, on ne manquera pas d'être surpris par l'absence de ces vers dans les sources et autres cours d'eau de surface des Mts. Apuseni et des Carpates Orientales, zones ayant cependant fait l'objet de nombreuses recherches et d'où les habitats propices ne font nullement défaut; ensuite, la présence de certaines populations (espèces?) à plus de $1000 \mathrm{~m}$ d'altitude dans les Carpates $(\mathrm{XV}, \mathrm{XVI})$, est une preuve du caractère actuellement expansif du groupe.

2. J'avais déjà signalé (Botosaneanu, 1971) le fait que les Tricladides dépigmentés et aveugles peuplent une multitude d'habitats du domaine aquatique souterrain, qu'en général chaque espèce est inféodée à un certain type d'habitat, et qu'il n'y a rien de plus faux et qui puisse donner une image plus appauvrie de la réalité, que considérer toujours ces animaux trouvés dans des eaux épigées comme éléments hypogés accidentellement entraînés dans celles-ci et pouvant y subsister dans certaines conditions. L'observation attentive de l'habitat et des ,,conditions de gisement" des animaux, suffit d'habitude pour trancher dans chaque cas.

Dans le présent travail, je présente des cas de formes peuplant le Karstostygal* ou les eaux de conduits et fissures (XVII-XIX); le Eustygal ou les nappes phréatiques proprement dites (XXII; X - mais peut-être s'agit-il, dans ce cas,

\footnotetext{
* Terminologie partiellement empruntée aux travaux de S. Husmann, partiellement inspirée par ceux-ci; voir Botosaneanu, 1971 (pp. 151 et suivantes, ainsi que la Bibliographie).
} 
de Pedostygal - "biotope hypotelminorhéique" de M. Mestrov - "nappes éluviales de déversement" des auteurs français; XVI et XX - avec affleurement dans des sources); le Rhythrostygal ou biotope hyporhéique (IV, V, VIII); le Crénal et le Rhythral, donc des sources et des ruisselets de source (I, VI, VII, IX, XII-XIV, XV, XXI); tous ces cas ne sont pas en égale mesure concluants); et même les niches madicoles ou hygropétriques (II).

Il serait intéressant de pouvoir reconstituer la séquence des événements ayant conduit les espèces des bassins lacustres en voie d'évanouissement vers la fin du Néogène, à coloniser le domaine aquatique souterrain et ses affleurements (donc la séquence des habitats colonisés). A mon avis, le schéma le plus en accord avec les faits connus serait le suivant:

LIMNOSTYGAL $\rightarrow$ RHYTHROSTYGAL

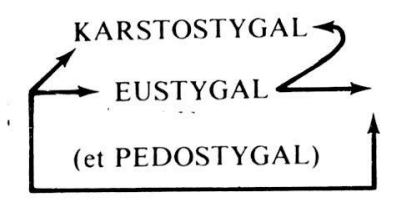

CRENAL et RHYTHRAL

Lorsque toutes les espèces seront décrites, leurs habitats et leurs affinités connus, ce schéma pourrait subir des modifications. Ce qui me semble hors de doute, c'est que le rôle des milieux interstitiels (Limnostygal, R hythrostygal) a été primordial et décisif dans ce processus.

3. Animaux de complexité très délicate mais carnivores, toujours lucifuges, psychrosténothermes et sténotopes, les Tricladides dépigmentés et aveugles sont des indicateurs extrêmement sensibles des conditions abiotiques et des relations biotiques régnant dans leurs habitats, et réagissent promptement même à des changements mineurs de ces conditions. Ceci devrait expliquer leur stricte localisation dans certains endroits d'une certaine station (VII, X, XII, XVI, $\mathrm{XX}, \mathrm{XXI}$ ), ainsi que leur absence d'habitats en apparence fort propices à leur installation, et situés au voisinage immédiat de stations où des populations de Tricladides obscuricoles existent (VII, XI, XII, XIII, XX). L'exclusion compétitive avec les Tricladides épigés, pigmentés, est le cas pratiquement général, cette exclusion prenant parfois des aspects fort incisifs; des cas de coexistence m'ont toutefois été signalés, mais ceux que je rapporte ici (III, VI, XI) devraient être soigneusement revus. En Roumanie, deux ou plusieurs espèces de Tricladides obscuricoles n'ont jamais été trouvées dans la même station. Les cas de coexistence avec d'autres animaux obscuricoles sont, bien sûr, assez nombreux (I, X, XII, XVI, XVII-XIX, XXII). J'ai fait des observations sur ce qui représente certainement des rapports de concurrence avec des Amphipodes épigés lorsque ceux-ci atteignent une trop grande densité (VII) et peut-être aussi avec des Niphargus (XVI). La fragilité de ces animaux et la labilité de leurs relations avec des habitats souvent très fragiles, ressortent clairement des cas observés de modifications anthropogènes de ces derniers, pouvant avoir un 
impact considérable sur leurs populations de Tricladides obscuricoles (IV, V, VI?, VIII, XII, XVI, XX, XXI); voir à ce sujet aussi le travail de Botosaneanu, 1972. En effet, on dirait qu'ils sont maudits, ces habitats d'une fraction des plus intéressantes de la faune stygobie: inondés par l'eau des lacs de barrage, anéantis par l'exploitation des carrières ou des alluvions, piétinés par les touristes ou par le bétail, captés et diversement modifiés s'il s'agit de sources.

\section{RESUME}

Vingt-deux localités, où ont été découverts par l'auteur lui-même des Turbellariés Tricladides (Dendrocoelidae et Fonticola) dépigmentés et le plus souvent aveugles, sont décrites avec plus ou moins de détails. Ces animaux sont particulièrement bien représentés en Roumanie. Cette situation s'explique par le fait qu'ils sont les descendants d'une faune ayant habité autrefois les immenses lacs saumâtres et d'eau douce qui recouvraient la plus grande partie de cette région au Néogène (et surtout au Sarmatien). Différentes espèces sont devenues les hôtes des divers habitats propres au domaine des eaux souterraines et l'auteur fait une distinction entre espèces vivant dans les eaux des grottes, phréatobiontes typique, espèces hyporhéiques et espèces vivant dans les sources ou les ruisseaux. Ces espèces sont des indicateurs sensibles des changements, même minimes, qui affectent les conditions abiotiques ou biotiques régnant dans leurs habitats (de nombreux exemples sont présentés, particulièrement d'exclusion compétitive).

\section{BIBLIOGRAPHIE}

BOTOSANEANU, L. 1971. Observations sur la faune aquatique hypogée des Monts du Banat (Roumanie). Trav. Inst. Spéol. “Emile Racovitza”, 10, 123-166.

BOTOSANEANU, L. 1972. Protection de la faune des eaux souterraines de Roumanie (en roumain). Ocrotirea Naturii, 16, 43-46.

BOTOSANEANU, L., NEGREA, A. et NEGREA, St. 1966. Grottes du Banat explorées de 1960 à 1962. In: Recherches sur les grottes du Banat et d'Olténie. Editions du CNRS, Paris.

CODREANU, R. et BALCESCO, DOINA, 1967. Sur trois Dendrocoelides aveugles nouveaux des sources du Barnt (Roumanie). Rev. Roum. Biol.-Zoologie, 12, 287-294.

CODREANU, R., et BA ESCO, DOINA. 1967. Sur les rapports entre les sous-genres Paradendrocoelum Kenk 1930 et Dendrocoelides De Beauchamp 1919 d'après les espèces obscuricoles du Banat et de l'Olténie. Rev. Roum. Biol.-Zoologie, 12, 337-349.

CODREANU, R. et BALCESCO, DOINA. 1970. Répartition des Dendrocoelides anophtalmes dans les Carpates de Courbure et dans la Plaine Roumaine. Livre du centenaire E. G. Racovitza. Editions de l'Académie de la R. S. Roumanie, Bucarest.

DEL PAPA, R. 1965. Descrizione di Dendrocoelum (Eudendrocoelum) Botosaneanii N. sp. delle grotte del Banato (Romania). Monit. Zool. It., 73, 156-162.

DEL PAPA, R. 1970. Su Dendrocoelum (Eudendrocoelum) botosaneanui mihi delle grotte del Banato (Romania). Zool. Anz., 184, 229-232.

GOURBAULT, NICOLE. 1967. Triclades obscuricoles de Roumanie. I. Dendrocoelides du Banat. Bull. Mus. National Hist. Nat. (2e Série), 39, 745-752.

SAULEA, EMILIA (coordunnateur). 1969. Atlas lithofacial (Roumanie). VI - Néogène. Institut Géologique, Bucarest. 